\title{
A novel practical approach for the form finding of the prism tensegrity
}

\author{
Mohammad Moghaddas ${ }^{1, *}$ Kok Keong CHOONG $^{2}$ \\ ${ }^{1,2}$ School of Civil Engineering, University of Science Malaysia, Penang, Malaysia \\ mohamad.moghadas@gmail.com
}

\begin{abstract}
The prism tensegrity is one of the types of the tensegrity system which due to its special characteristics, can cover all possible application of tensegrity in the field of civil and the structure engineering. According to the other researches and based on the process of the available form finding approaches, it is concuded that there is no practical approach for the form finding of the irregular prism tensegrity with a large number of members. In this paper a novel linear approach is introduced which can be used as a practical approach for the form finding of irregular prism tensegrity.
\end{abstract}

Keywords: Prism tensegrity, Form finding, Irregular shape, linear approach

\section{INTRODUCTION}

The word tensegrity for the first time was used by R. Buckminster Fuller in 1961. He coined the word from tension and integrity [1]. The origin of tensegrity, however, is the most peculiar and controversial aspect of it. Despite the existing claims and patents, the talk on the origin of tensegrity is still polemical. [2]. One of these patents with the name of "Tensile Integrity" was proposed by R. Buckminster Fuller in 1959 and granted in 1962 [3]. Another patent belongs to David Georges Emmerich. His firstly proposed "Pearl Frameworks" patent in 1959, unsuccessful attempt. He proposed the second one named as "Constructionde rdseaux autotendants" in 1963 and it was granted in 1964. The last patent with the name of "Continuous tension, discontinuous compression structures" belongs to Kenneth Snelson. It was filed in 1960 and granted in 1965 [4].

Tensegrity system serves a broad range of applications. It applies in the fields such as life sciences, engineering, and architecture, or in an entirely different area such as art. In life sciences, the tensegrity system is used to model a human skeleton or cytoskeleton [5, 6]. In art, it is used to create sculpture and ornamental furniture [7]. Robots and wings of aircraft are the two examples of the application of tensegrity system in the field of mechanical engineering [810]. Tensegrity system also applies in the field of aerospace [11]. It has been used in the construction of antennas for satellites $[12,13]$. In addition tensegrity system can be engaged for construction of space station [14].

The other application area of tensegrity system is in the field of civil and construction engineering $[15,16]$. The tensegrity system is used in the construction of the bridges $[17,18]$, shelter and tent [19]. Figure 1 shows a bridge and shelter based on tensegrity system. In addition, some future construction can be built based on tensegrity system. The huge dome over cities for environmental control, energy transformation and food production is one of the many futures application of tensegrity system. Large scale shielding for lightening, electromagnetic 
and micrometeorite protection is another future usage of tensegrity. Through the tensegrity system, containment or quarantine over large areas to hold flying birs, and new residential areas in rugged environments like Mars or under the sea will also be practical.
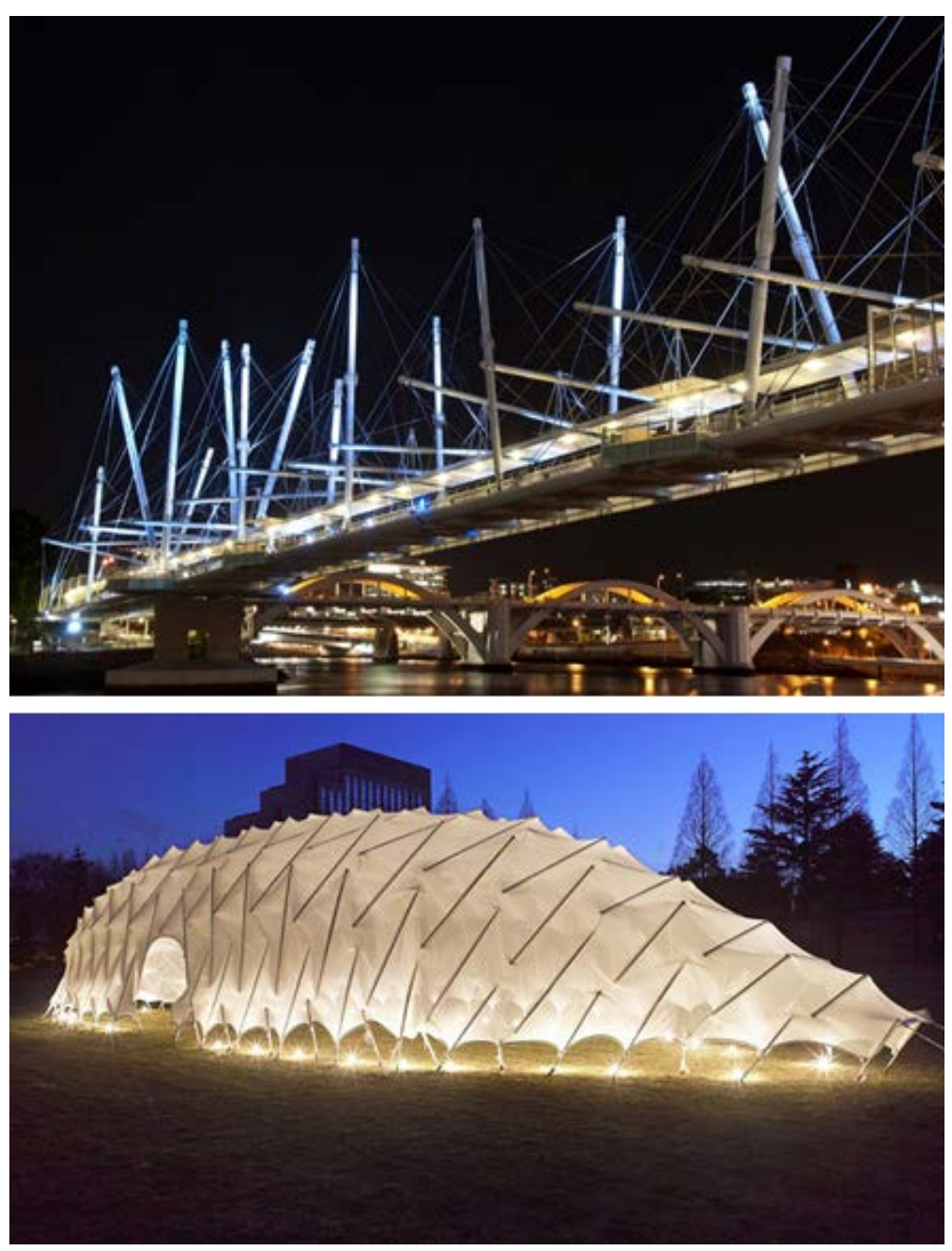

Figure 1: The bridge (top figure) and the shelter (bottom figure) based on tensegrity system

All the tensegrity systems are classified by the relative in the members into the four main groups of Rhombic (prism), Zigzag, Star, and Circuit [20]. Figure 2 depicts a basic cell of these systems. The circuit, the zigzag, and the star patterns are achievable through modification of prism tensegrity system [21]. Therefore, the prism system is the base for the all tensegrity systems. 

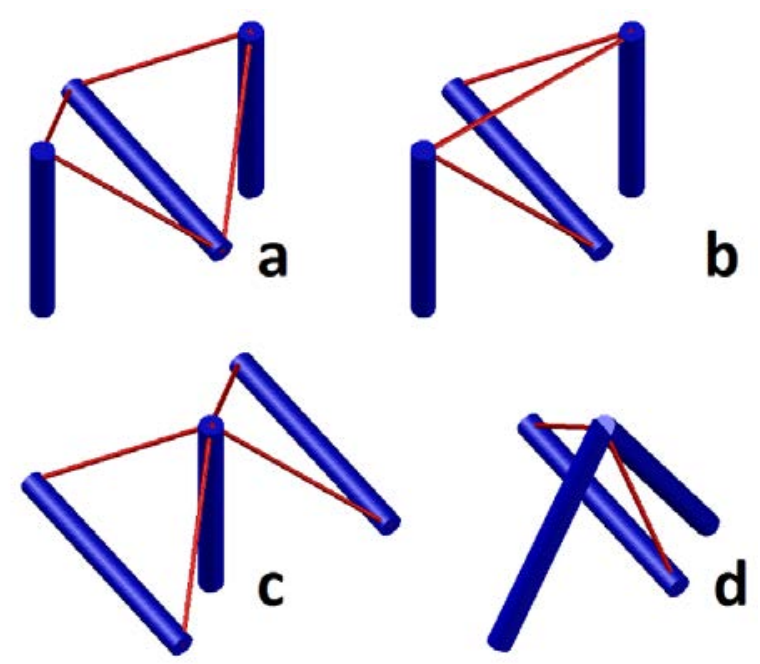

Figure 2: a: Prism tensegrity cell, b: Zigzag tensegrity cell, c: Star like tensegrity cell, d: Circuit tensegrity cell

The process of finding the geometric self-equilibrium shape of tensegrity system are known as form finding. During the form finding process, the lengths of the members and the associated axial force are determined [22]. Numerous the form finding approaches have been introduced for finding of the geometric shape of tensegrity system.Fuller and Snelson began to study the tensegrity system to determine of the geometrical configuration $[3,4,23]$. Then the other researchers presented some analytical or numerical methods and approaches for the form finding of the tensegrity system. Tiber and pllegrino divided the existing approaches into the two main groups of kinematical and statically [24]. They concluded that the analytical approaches by using the geometric properties and the force equilibrium equation are feasible for a small system with limited variations or a highly symmetrical system. Also the nonlinear general approach and the dynamic relaxation can only be applied to the known configuration with the identified coordinates. They proved that the potential energy approach and the force density approach are linked and equivalent. They commented the intuitive method to find the force density matrix is only suitable for a system with few members. The iterative one is a trial and error procedure which is not practical for a large system. They employed the analytical method to find the force density matrix to solve some examples, however, the outcomes of this method was unpredictable. Therefore, it is not suitable for finding the desired length of members and configurations. According to Tibert and Pellegrino's opinion, the reduce coordinate method controls the configuration. However, in the case of irregular tensegrity or a system with a large number of members, it requires a huge symbolic equations to be solved which is practicably not possible.

Recently many research studies on the form finding of the tensegrity system were carried out, but somehow their presented methods are linked to one of the previous approaches. Masic and his colleagues simplified the force density method and reduced different variations by inputting the symmetrical consideration in the calculation process [25]. A linear direct approach for the symmetrical tensegrity structure was introduced by Zhang et al. [26]. Baudriller and his collegues proposed a real-time processing solution for the form finding of the irregular tensegrity system based on the dynamic relaxation approach [27]. Pagirz and Tur used the finite element method to improve the minimization potential energy approach [28]. Koohestani and Guest extended the force density approach by benefiting from the Cartesian components of the members directly instead of their length in the calculation [29]. 
In a prism tensegrity structure, the number of equilibrium equations that can be written for a prism tensegrity system is equal to the number of its joints. Only the force equilibrium equation alone is not sufficient for the form finding of a prism tensegrity without using extra condition such as symmetrical. Otherwise, the calculations of the form finding became nonlinear and it requires a trial and error method to solve the equations. With respect to the number of variations in an irregular prism tensegrity system, an approach with nonlinear calculations is only feasible for an unsymmetrical structure with a few members. Otherwise, the number of loops of trial and error for converging are unlimited and practically is unsolvable.

Considering the previous research works and the available approaches, it is concluded that there is a need for the practical approach based on the linear equations for the irregular prism tensegrity. In this research, a novel linear approach is introduced particularly for the form finding of the symmetrical and the unsymmetrical prism tensegrity system including those with a large number of members. Furthermore, this novel approach is practical because it is highly accurate, fast in the calculation, and designers have full control on the results for desired configurations.

\section{FORM FINDING OF PRISM TENSEGRITY STRUCTURE}

The prism tensegrity system consists of two polygons in two parallel planes. It is assumed that (xy) plane is parallel to the two polygons while the $z$-axis is perpendicular to this plane. The lower polygon is called the first ring and the upper one is called the second ring. In this study, the first polygon is always the lower one and the second one is the upper polygon for positive $z$. The number of vertices is symbolized as $n$ and they are the same in both lower and upper polygons. In the following descriptions, $i$ stands for a particular joint where $i \in(1-n) \cdot j_{i}$ and ${ }_{j}{ }_{i}$ represent the joints of the first ring and the second ring, respectively. Figure 3 shows the six joints of the first and the second polygon with their connected members.

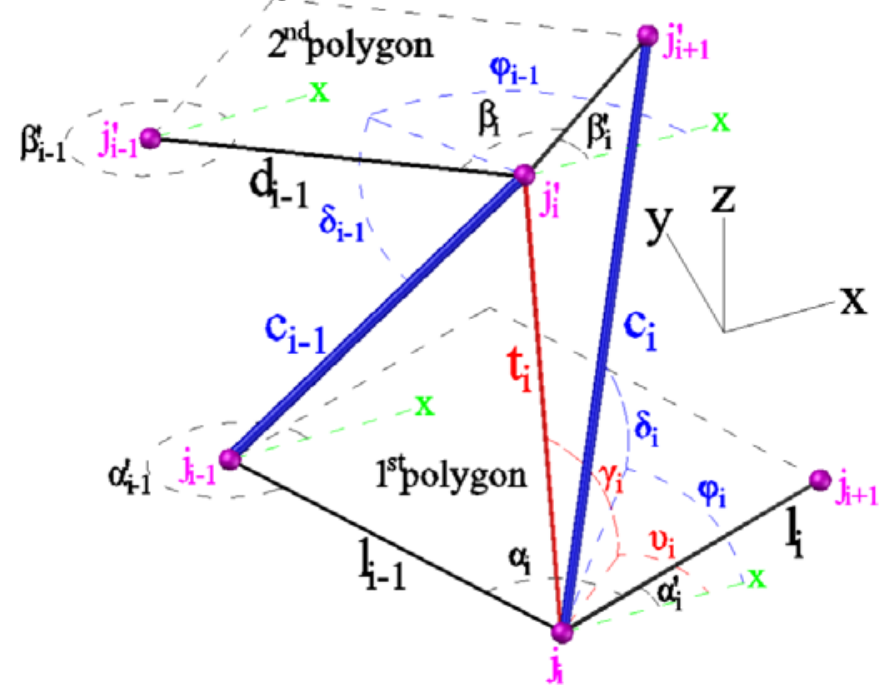

Figure 3: The six joints of the first and second ring with their connected members

In Figure 3, the internal angle of vertices in the first polygon is shown as $\alpha_{i}$ and the internal angles of the second ring is denoted as $\beta_{i}$. The symbol for members of the first ring is $l_{i}$. In the first ring, $j_{i}$ and $j^{\prime}{ }_{i}$ are the first and second joints of member $l_{i}$, respectively. The members of the second ring are indicated by $d_{i} \cdot j^{\prime}{ }_{i}$ and $j^{\prime}{ }_{i+1}$ are the first and second joints of the member $d_{i}$, respectively. The angles between $x$-axis and initial joint of $l_{i}$ and $d_{i}$ are denoted respectively as 
$\alpha^{\prime}{ }_{i}$ and $\beta_{i}$. This angle is generally known as azimuth angle. The tension diagonal members are symbolized by $t_{i}$ and $c_{i}$ is nominated as compression diagonal members. The two ends of the member $t_{i}$ are $j_{i}$ and $j^{\prime}{ }_{i}$. Also, the $j^{\prime}{ }_{i}$ and $j_{i+1}$ are two end joints of $d_{i}$. The angle between a diagonal members and ( $x y)$ plate is commonly called the polar angle. This angle is denoted as $\delta_{i}$ for compression diagonal members and $\gamma_{i}$ for tension diagonal members. The $\varphi_{i}$ and $v_{i}$ represent the azimuth angle of $d_{i}$ and $l_{i}$, respectively.

In the multi-layer prism tensegrity, the number of joint in the first ring of the first layer is specified the number of joint in the polygons of another layer. Also, in a prism tensegrity, the number of joints of the second ring is equal to the number of the joint of the first ring. Therefore, the first ring of the first layer is nominated as the base polygon.

In the first step of this novel approach, a closed polygon should be specified by the designer as which meets all the requirements of the project and available condition. This assumed polygon is the base ring of the prism tensegrity. Unlike other methods that designer should assume the topology of the whole system at the first, the topology is prism tensegrity system and determined in this approach. There is no limitation on the number of the joints and configuration of the assumed base polygon except that all its internal angles must be converge. Subsequent to the determination of the first polygon, based on the description of prism tensegrity structure, the number of vertices and the plane of the second polygon in space is defined. But this information is not enough for defining a particular polygon for the second ring and there is an unlimited possible combination for the second polygon. Therefore, to find a specific second polygon according to the preference of designer, the orientations of two subsequent members of the second polygon is required. Moreover, the vertex between these two members of the second polygon, is connected to a joint in the first ring which determined by the designer. For simplification, this vertex is supposed as the first joint of the second ring. In the first ring, the joint which is connected to the first joint of the second ring by a diagonal tension member is assumed as the first joint of the first ring.

The parameters which should be defined by designer for using the novel approach are:

$l_{1}, l_{2}, l_{3}, \ldots, l_{n} ;\left\{\alpha_{1}, \alpha_{2}, \propto_{3}, \ldots, \propto_{n}\right\}<\pi ; \propto_{1}^{\prime}, \propto_{2}^{\prime}, \propto^{\prime}{ }_{3}, \ldots, \propto_{n}^{\prime} ; \beta_{n}^{\prime}, \beta_{1}^{\prime}, \beta_{1}$

These parameters are the specified first polygon and orientations of two subsequent members of the second polygon. In the proposed novel approach, a large number of parameters is to be specified by the designer. This attribute increases the flexibility of the design process to achieve a configuration based on the needs of designers.

To obtain a specified prism tensegrity structure, the unknown parameters are:

$d_{1}, d_{2}, d_{3}, \ldots, d_{n} ; \quad\left\{\beta_{2}, \beta_{3}, \beta_{4}, \ldots, \beta_{n}\right\}<\pi ; \quad \bar{F}_{l_{1}}, \bar{F}_{l_{2}}, \bar{F}_{l_{3}}, \ldots, \bar{F}_{l_{n}} ; \quad \bar{F}_{d_{1}}, \bar{F}_{d_{2}}, \bar{F}_{d_{3}}, \ldots, \bar{F}_{d_{n}} ;$ $\bar{F}_{t_{1}}, \bar{F}_{t_{2}}, \bar{F}_{t_{3}}, \ldots, \bar{F}_{t_{n}} ; \bar{F}_{c_{1}}, \bar{F}_{c_{2}}, \bar{F}_{c_{3}}, \ldots, \bar{F}_{c_{n}}$

These parameters should be calculated by using a form finding approach based on the assumptions of the designer.

Figure 4 Shows the step by stem flow chart of the novel approach for the form finding of the irregular or regular prism tensegrity with any number of members. Moreover, Equation (1) to (6) present the required equations of this flowchart. 
Bulletin de la Société Royale des Sciences de Liège, Vol. 85, 2016, p. 1360 -

$$
\begin{aligned}
& \frac{\bar{F}_{d_{i}}}{\sin \left(\beta_{i-1}^{\prime}-\alpha_{i-1}^{\prime}\right)}=\frac{\bar{F}_{d_{i-1}}}{\sin \left(\alpha_{i-1}^{\prime}+\beta_{i}-\beta_{i-1}^{\prime}\right)}=\frac{k \cdot \bar{L}_{l_{i-1}}}{\sin \beta_{i}} \\
& \bar{F}_{d_{i}}=\sqrt{\bar{L}_{l_{i-1}}{ }^{2}+\bar{F}_{d_{i-1}}{ }^{2}-2 \bar{L}_{l_{i-1}} \bar{F}_{l_{i-1}} \cos \left(\beta^{\prime}{ }_{i-1}-\alpha^{\prime}{ }_{i-1}\right)} \\
& \frac{\bar{F}_{l_{i}}}{\sin \left(180+\alpha^{\prime}{ }_{i-1}-\beta_{i}^{\prime}\right)}=\frac{\bar{F}_{l_{i-1}}}{\sin \left(\alpha^{\prime}{ }_{i-1}-\beta_{i}^{\prime}-\alpha_{i}\right)}=\frac{k^{\prime} \cdot \bar{L}_{d_{i}}}{\sin \alpha_{i}} \\
& \bar{L}_{d_{i}}=\sqrt{\bar{F}_{l_{i}}^{2}+\bar{F}_{l_{i-1}}{ }^{2}-2 \bar{F}_{l_{i}} \bar{F}_{l_{i-1}} \cos \alpha_{i}} \\
& \bar{F}_{l_{i}}=\sqrt{\bar{F}_{l_{i-1}}{ }^{2}+\bar{L}_{d_{i}}{ }^{2}-2 \bar{F}_{l_{i-1}} \bar{L}_{d_{i}} \cos \left(\beta_{i}^{\prime}-\alpha^{\prime}{ }_{i-1}\right)} \\
& \frac{\bar{L}_{l_{i-1}}}{\sin \left(\varphi_{i-1}-\vartheta_{i}\right)}=\frac{\bar{F}_{t_{i}} \cos \gamma_{i}}{\sin \left(\varphi_{i-1}-\alpha^{\prime}{ }_{i-1}\right)}=\frac{\bar{F}_{c_{i-1}} \cos \delta_{i}}{\sin \left(180+\alpha^{\prime}{ }_{i-1}-\vartheta_{i}\right)}
\end{aligned}
$$


Bulletin de la Société Royale des Sciences de Liège, Vol. 85, 2016, p. 1360 -

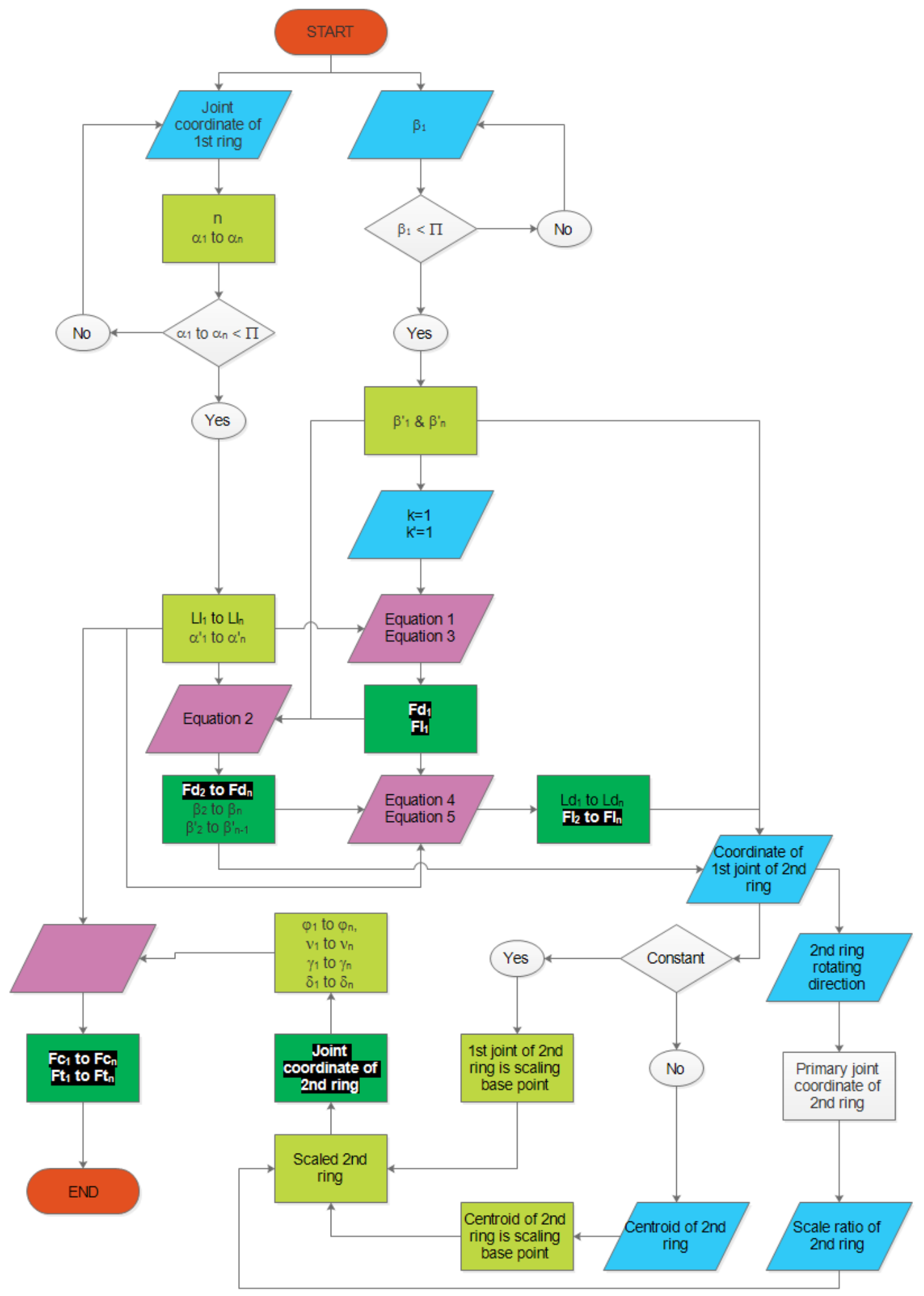

Figure 4: The flow chart of trigonometry approach for the form finding of the prism tensegrity 


\section{NUMERICAL EXAMPLES}

\subsection{Example 1}

This example is a symmetrical prism tensegrity with 12 members and 6 joints. The input data for the computer tool based on the new approach and outputs are shown in Figure 5. Moreover Figure 6 illustrates the 3D view of the final configuration.

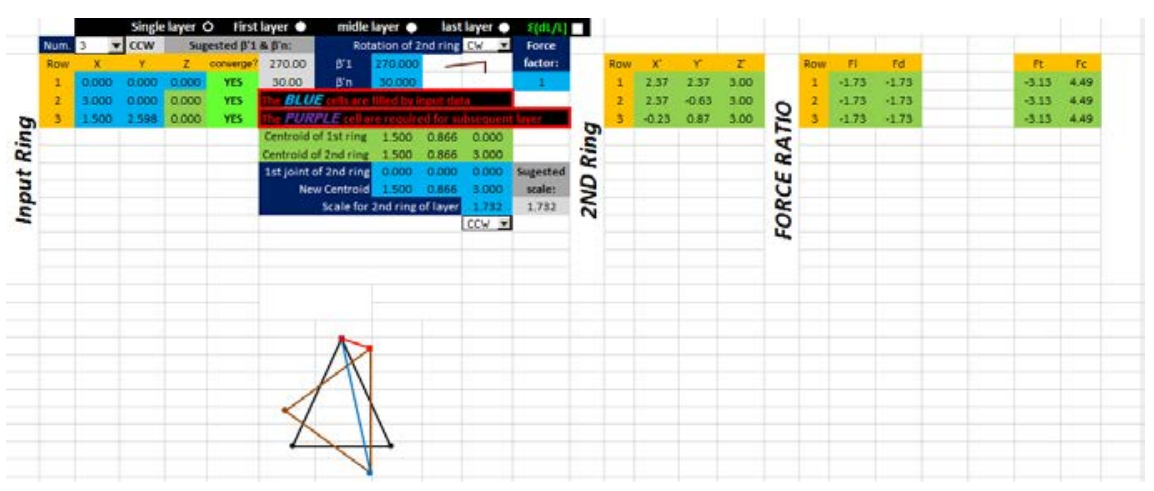

Figure 5: The input and the output of the first example

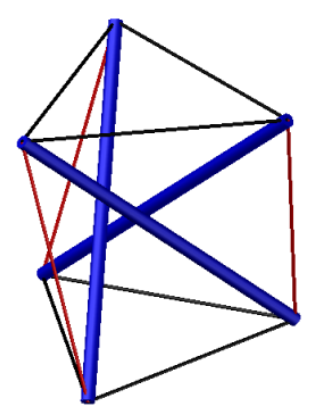

Figure 6: The 3D view of the first example

\subsection{Example 2}

This example is an unsymetrical prism tensegrity with 20 joints and 40 members. It is assumed based on the desiere of the designer, the length and the direction of the firs member of the second ring should be equal to the second member of the first ring. The view from inputs and outputs of the computer tools is presented in Figure 7. Figure 8 shows the 3D view from the final shape.

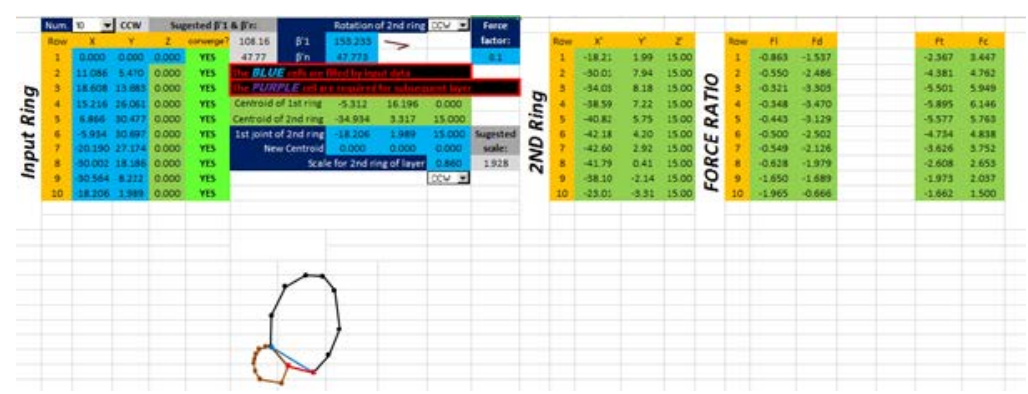

Figure 7: The input and the output of the second example 


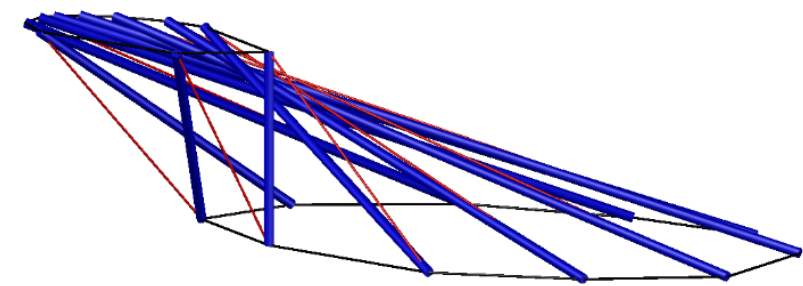

Figure 8:The 3D view of the second example

\subsection{Example 3}

In the Figure 9 and Figure 10, a complex prism tensegrity structure which is designed by this new approach are presented to exhibit the ability of this approach. This example has 800 joints and 1600 members. Moreover, the joints create 58 unsymmetrical unique rings. It is not possible to design this example by any other available approach. Figure 9 and Figure 10 illustrate respectivly two different kind of the 3D and front views from the final configuration of this example.

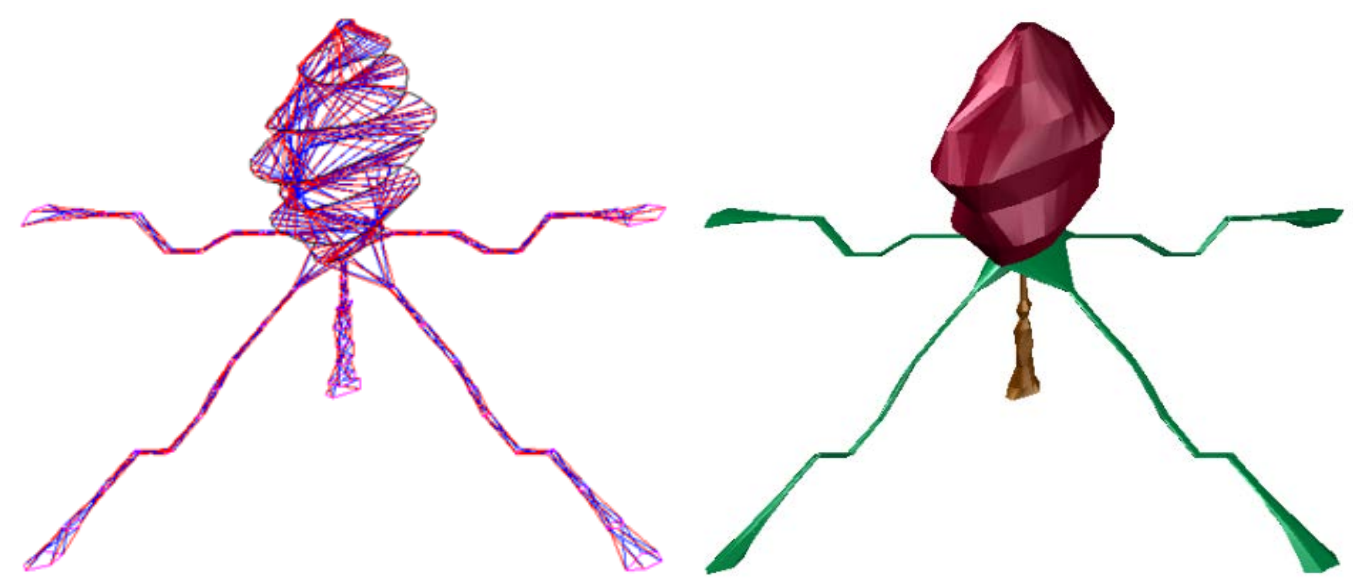

Figure 9: The normal and covered 3D views of the complex multi-layer prism tensegrity

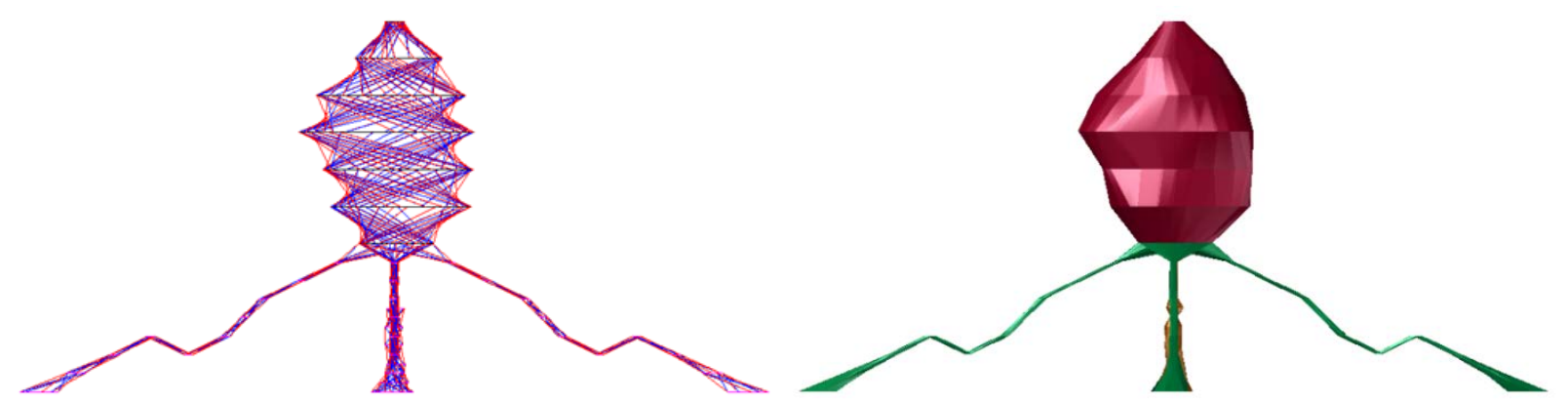

Figure 10: The normal and covered front views of the complex multi-layer prism tensegrity

\section{CONCLUSION}

In this research, the drawback of the current form finding approaches for different tensegrity system was unveiled. The most critical drawback of other approaches is the used nonlinear 
equation in the calculation. However, solving nonlinear equations requires symmetrical conditions or is achievable through trial and error which makes it incompatible with the irregular structures. In the current study, a novel practical approach is presented for form finding of regular or irregular prism tensegrity system with any numbers of members. This novel approach is linear and it does not require any additional condition or iteration of trial and error. Therefore, this approach is compatible with irregular or regular prism tensegrity with a large number of members.

A computer tool was developed based on the novel approach. Since the presented approach is linear, the computer tool runs fast and immediately responses to any changes in the input and the changes are detectable in the final result. The results of this software are the force ratio of members, the strain of members and output for direct modeling in Autodesk AutoCAD.

\section{REFERENCES}

[1] Fuller, B., Tensegrity. Portfolio and Art News Annual. 1961. p.

[2] Jáuregui, V.G., Tensegrity structures and their application to architecture, PUbliCan. Vol. 2010.

[3] Fuller, R.B., Tensile-integrity structures, United States Patent 3,063,521. 1962. p.

[4] Snelson, K.D., Continuous tension, discontinuous compression structures, United States Patent 3,169,611. 1965. p.

[5] Aranda-Anzaldo, A., The interphase mammalian chromosome as a structural system based on tensegrity. Journal of theoretical biology, 2016. p.

[6] Sheng, J., A Computational Model of Cell Movement Linked to Substrate Rigidity, Lehigh University. Vol. 2015.

[7] Hu, N., P. Feng, and G.-L. Dai, Structural art: Past, present and future. Engineering Structures, 2014. 79: p. 407-416.

[8] SunSpiral, V., A. Agogino, and D. Atkinson, Super Ball Bot-Structures for Planetary Landing and Exploration, NIAC Phase 2 Final Report. 2015. p.

[9] Lian, O.C., C.K. Keong, and L.C. Yee, Biotensegrity Inspired Robot-Future Construction Alternative. Procedia Engineering, 2012. 41: p. 1079-1084.

[10] Bart-Smith, H., et al. Optimization of a tensegrity wing for biomimetic applications. in Decision and Control, 2006 45th IEEE Conference on. IEEE. 2006.

[11] SunSpiral, V., et al., Tensegrity based probes for planetary exploration: Entry, descent and landing (EDL) and surface mobility analysis. International Journal of Planetary Probes, 2013. p.

[12] Tibert, G., Deployable tensegrity structures for space applications, Royal Institute of Technology. Vol. 2002.

[13] Puig, L., A. Barton, and N. Rando, A review on large deployable structures for astrophysics missions. Acta Astronautica, 2010. 67(1): p. 12-26.

[14] Murata, S., et al. Inflatable Tensegrity Module for a Large-Scale Space Structure and its Construction Scinario. in The 56th International Astronautical Congress (IAC05). 2005.

[15] Pedretti, M. Smart tensegrity structures for the Swiss Expo 2001. in 5th Annual International Symposium on Smart Structures and Materials. International Society for Optics and Photonics. 1998.

[16] Gilewski, W., J. Kłosowska, and P. Obara, Applications of Tensegrity Structures in Civil Engineering. Procedia Engineering, 2015. 111: p. 242-248. 
[17] Veuve, N., S. Dalil Safaei, and I.F.C. Smith, Active control for mid-span connection of a deployable tensegrity footbridge. Engineering Structures, 2016. 112: p. 245-255.

[18] Skelton, R.E., et al., Minimum mass design of tensegrity bridges with parametric architecture and multiscale complexity. Mechanics Research Communications, 2014. 58: p. 124-132.

[19] Schofield, C.C., PORTABLE TENSEGRITY STRUCTURE, US Patent 20,150,330,098. 2015. p.

[20] Pugh, A., An introduction to tensegrity, Univ of California Press. Vol. 1976.

[21] Motro, R., Tensegrity: structural systems for the future, Elsevier. Vol. 2003.

[22] Skelton, R.E., et al. An introduction to the mechanics of tensegrity structures. in Decision and Control, 2001. Proceedings of the 40th IEEE Conference on. IEEE. 2001.

[23] Fuller, R.B., Marks, R., THE DYMAXION WORLD OF BUCKMINSTER FULLER, Reinhold Publications, New York. 1960. p.

[24] Tibert, A.G. and S. Pellegrino. Deployable tensegrity masts. 2003.

[25] Masic, M., R.E. Skelton, and P.E. Gill, Algebraic tensegrity form-finding. International Journal of Solids and Structures, 2005. 42(16): p. 4833-4858.

[26] Zhang, J., M. Ohsaki, and Y. Kanno, A direct approach to design of geometry and forces of tensegrity systems. International Journal of Solids and Structures, 2006. 43(7): p. 2260-2278.

[27] Baudriller, H., et al., Form-finding of complex tensegrity structures: application to cell cytoskeleton modelling. Comptes rendus mécanique, 2006. 334(11): p. 662-668.

[28] Pagitz, M. and J.M. Tur, Finite element based form-finding algorithm for tensegrity structures. International Journal of Solids and Structures, 2009. 46(17): p. 3235-3240.

[29] Koohestani, K. and S.D. Guest, A new approach to the analytical and numerical formfinding of tensegrity structures. International Journal of Solids and Structures, 2013. 50(19): p. 2995-3007. 